\title{
The benefit-to-risk ratio of common treatments in PCOS: effect of oral contraceptives versus metformin on atherogenic markers
}

\author{
Charikleia Christakou, ${ }^{1}$ Anastasios Kollias, ${ }^{1}$ Christina Piperi, ${ }^{2}$ Ilias Katsikis, ${ }^{3}$ \\ Dimitrios Panidis, ${ }^{3}$ Evanthia Diamanti-Kandarakis ${ }^{1}$
}

${ }^{1}$ Endocrine Unit, Third Department of Internal Medicine, 'Sotiria' Hospital; ${ }^{2}$ Department of Biological Chemistry, Medical School; University of Athens; Athens; ${ }^{3}$ Division of Endocrinology and Human Reproduction, Second Department of Obstetrics and Gynecology, Medical School, Aristotle University of Thessaloniki, Thessaloniki; Greece

\begin{abstract}
OBJECTIVE: To compare the effects of oral contraceptives (OCPs) and metformin on atherogenic markers, including serum levels of advanced glycated end products (AGEs) and Creactive protein (CRP), in lean women (Body Mass Index below $25 \mathrm{~kg} / \mathrm{m}^{2}$ ) with polycystic ovary syndrome (PCOS), defined by NIH criteria. DESIGN: Prospective open-label study. RESULTS: One hundred and twenty women with PCOS were treated for 6 months with one of the following treatments: ethinylestradiol plus cyproterone acetate $(\mathrm{OCP} 1, \mathrm{n}=40)$ or ethinylestradiol plus drospirenone $(\mathrm{OCP} 2, \mathbf{n}=40)$ or metformin $(\mathrm{MET}, \mathrm{n}=40)$. The three groups were age and BMI-matched (mean age: $22 \pm 0.56 \mathrm{yrs}$ in group OCP1; $23.24 \pm 0.64 \mathrm{yrs}$ in group OCP2; 21.50 \pm 0.53 yrs in group MET; mean BMI $21.80 \pm 0.35 \mathrm{~kg} / \mathrm{m}^{2}$ in group $O C P 1 ; 22.37 \pm 0.48 \mathrm{~kg} / \mathrm{m}^{2}$ in group OCP2; $23.03 \pm 0.67 \mathrm{~kg} / \mathrm{m}^{2}$ in group MET). At 6 months serum AGEs were decreased in group OCP1 $(P=0.005)$ and group MET $(P=0.001)$, whereas these were marginally decreased in group $\mathrm{OCP} 2(\mathrm{P}=0.069)$. Treatment with metformin was associated with a greater percent decrease of AGEs. CRP was decreased with metformin $(P<0.001)$, but was increased with OCPs $(P<0.001)$. CONCLUSIONS: This study evaluates common therapeutic options in women with PCOS by reconsidering and prioritizing the goals of treatment. OCPs and metformin appear to have differential effects on atherogenic molecules in lean PCOS patients, but metformin was superior in reducing serum AGEs and CRP. Clinicians should individualize the benefit-to-risk ratio of pharmaceutical intervention in women with PCOS in order to choose the formulation with the greatest overall efficacy as well as safety in terms of cardiovascular risk.
\end{abstract}

Key words: Atherogenic markers, Metformin, Oral contraceptives, Polycystic ovary syndrome

Address for correspondence:

Evanthia Diamanti-Kandarakis, University of Athens Medical School, 1A Zefyrou Str., Ekali 17562, Athens, Greece, Fax: +301 2108130031; E-mail: e.diamanti.kandarakis@gmail.com Received: 25-05-2013, Accepted: 18-07-2013

\section{INTRODUCTION}

Polycystic ovary syndrome (PCOS) is a heterogenous female endocrinopathy which is characterized by chronic anovulation and clinical or biochemical 
hyperandrogenemia as well as insulin resistance and a clustering of cardiovascular risk factors. ${ }^{1}$ Even in the absence of detectable metabolic abnormalities, lean PCOS women have increased circulating levels of advanced glycated end products (AGEs), which are known proinflammatory and proatherogenic molecules. ${ }^{2}$

AGEs are irreversible products of nonenzymatic glucose-protein reactions. These molecules exert a potent impact on vessels and other tissues not only in hyperglycemic states but also in euglycemic conditions with increased oxidative stress. ${ }^{3}$ Accumulation of AGEs has been demonstrated in several tissues, including the vasculature and the ovary. ${ }^{3,4}$ Thus, AGEs may play an important role in the pathogenesis of PCOS by contributing not only to metabolic but also to reproductive aspects.

Progress in understanding the syndrome's pathophysiology during the last two decades has led to reconsideration of its therapeutic management, which currently includes oral contraceptives (OCPs) and metformin. Although OCPs remain the mainstay of the pharmacological therapy of PCOS, even low dose pills may be metabolically less safe and are linked to an increased risk of myocardial infarction and stroke. ${ }^{5,6}$ These data have raised major concerns over the long-term safety of this treatment.

Metformin, the most widely prescribed insulin sensitizer, appears to improve metabolic aspects and cardiovascular risk factors in women with PCOS. In particular, metformin treatment for 6 months was shown to alleviate the proinflammatory status and reduce serum AGEs levels in women with PCOS. ${ }^{7,8}$

However, no previous study has compared different types of OCPs and metformin regarding their effects on serum AGEs in women with PCOS. The aim of the present study was to examine how two different types of OCPs and metformin affect serum AGEs and CRP in lean women with PCOS during 6 months of treatment.

\section{PATIENTS AND METHODS}

\section{Study population}

A total of 120 premenopausal Greek Caucasian women were recruited at the Outpatient Departments of Endocrinology of the two participant University Hospitals in Thessaloniki and in Athens, between 2008 and 2010, respectively.

All women visited the outpatient Departments because of hirsutism, menstrual abnormalities or subfertility. Their data were maintained prospectively in a computerized database. The ethics boards approved the study protocol and informed consents were obtained from all participants.

The diagnosis of PCOS was based on the presence of irregular menstrual cycles (eight or fewer menses per year) as well as elevated serum levels of testosterone and/or clinical symptoms of hyperandrogenism, according to the National Institute of Child Health and Human Development conference. ${ }^{9}$ Non-classical congenital adrenal hyperplasia, androgen-secreting neoplasms, hyperprolactinemia and thyroid disease were excluded by appropriate tests in all women. All the participants were lean (BMI $\left.<25 \mathrm{~kg} / \mathrm{m}^{2}\right)$, in good health and nonsmokers or had quit smoking for more than a year at baseline. Patients were excluded from participation if they were pregnant or planning to become pregnant, were breastfeeding, had a history of current or recent (within 6 months) use of oral contraceptives, antidiabetics, or antiandrogens, or had any contraindications to metformin therapy including renal or hepatic impairment.

\section{Study protocol}

E.D.-K. and D.P. enrolled the patients. Two doctors in each centre performed the baseline physical examination. Weight and height were measured in light clothing without shoes. Body mass index (BMI) was calculated as weight (kilograms)/height (meters). ${ }^{2}$ Patients were considered lean when their BMI was below $25 \mathrm{~kg} / \mathrm{m}^{2}$.

Waist-to-hip ratio (W/H ratio) was calculated as a marker of body fat distribution at baseline, according to the standard formula: WHR = waist circumference $(\mathrm{cm}) /$ hip circumference $(\mathrm{cm})$. Waist circumference was estimated as the smallest circumference at the level of the umbilicus. Hip circumference was estimated as the widest circumference at the level of the buttocks.

Baseline laboratory evaluation was conducted between the $2^{\text {nd }}-7^{\text {th }}$ day of the follicular phase of menstrual cycle confirmed by serum progesterone 
levels below $5 \mathrm{nmol} / \mathrm{l}$ or at any time in PCOS women with amenorrhea. Laboratory evaluation included metabolic and hormonal parameters, serum CRP and serum AGEs levels.

Blood samples were collected at 08:00 h after an overnight fast to determine serum levels of AGEs $(\mathrm{U} / \mathrm{ml})$, total testosterone (Testo, nmol/l), SHBG $(\mathrm{nmol} / \mathrm{l})$, fasting insulin $(\mathrm{pmol} / \mathrm{l})$ and glucose $(\mathrm{mmol} / \mathrm{l})$, $\Delta 4$-androstenedione (nmol/l) and dehydroepiandrosterone sulfate (DHEAS) (nmol/l). The free androgen index was calculated as follows: $\mathrm{FAI}=$ Testo $(\mathrm{nmol} / \mathrm{l})$ to SHBG (nmol/l) ratio x $100 \%$. Insulin resistance was assessed via calculation of the homeostasis model assessment insulin resistance index (HOMA-IR =fasting glucose in $\mathrm{mmol} / \mathrm{l} \mathrm{x}$ insulin in microunits per milliliter/22.5). Congenital adrenal hyperplasia was ruled out by evaluation of 17-hydroxyprogesterone and, when appropriate, ACTH stimulation test. If clinically suspected, screening for Cushing's syndrome was performed by the overnight dexamethasone suppression test (i.e. the measurement of a cortisol level the morning after the administration of $1 \mathrm{mg}$ dexamethasone orally at bedtime). An oral glucose tolerance test (OGTT) with 75-g GLU load was performed at baseline to exclude diabetes (times 0, 30, 60,90 , and 120 minutes) (data not provided). None of the patients was diabetic.

After completing baseline evaluation, patients were randomized in a 1:1:1 ratio to receive one of the following treatments for 6 months:

- Group OCP1 $(n=40)$ received the CPA pill containing $35 \mu \mathrm{g}$ ethinylestradiol and $2 \mathrm{mg}$ cyproterone acetate (Gynofen-35, Bayer),

- Group OCP2 $(n=40)$ received the DRSP pill containing $30 \mu \mathrm{g}$ ethinyl estradiol and $3 \mathrm{mg} \mathrm{dr}-$ ospirenone (Yasmin, Bayer),

- Group MET $(\mathrm{n}=40)$ received metformin (Glucophage; Group Lipha, Lyon, France).

Randomization was performed by random number tables. The patient number treatment codes were held by a third party.

Treatment was started on the first day of a spontaneous menstrual cycle. In amenorrhoeic women treatment was initiated on a random day after exclud- ing pregnancy by proper testing. $\mathrm{OCP} 1$ and $\mathrm{OCP} 2$ were administered in the recommended regimen (1 pill daily for 21 days per month, followed by a 7-day pill-free period). Metformin was given initially as 425 $\mathrm{mg}$ twice daily after meals for one week to minimize gastrointestinal upset. The dose was then increased to $850 \mathrm{mg}$ twice daily. Patients were instructed to continue their routine dietary and physical activity habits. Patients in group MET were instructed to use barrier methods of contraception.

Participants were re-evaluated at 3 months and 6 months of treatment. At each follow-up visit, physical examination and laboratory measurements (hormonal parameters, Glucose, Insulin, HOMA-IR, AGEs and CRP) were repeated. The percent changes of each parameter at 6 months of treatment (DBMI, DHOMA, DTESTO, DSHBG, DAGEs, DCRP, DFAI) were calculated as the ratio of the absolute change of the parameter from baseline to the baseline value. Additionally, the amount of medicine taken in the antecedent 3-month period was recorded by the investigators to assess adherence to treatment.

\section{Assays}

Blood samples were collected from all participants at baseline, 3 months and 6 months of treatment, and centrifuged and sera and plasma were stored at $-80^{\circ} \mathrm{C}$ prior to analysis.

Just prior to assay by competitive AGE-ELISA, sera were thawed and diluted 1:5 with dilution buffer [phosphate-buffered saline (PBS), 0.02\% Tween-20 and $1 \mathrm{mM}$ sodium azide (NaN3)]. AGE measurements were performed using the Chemwell analyser (Awareness, Florida, USA).

Plasma glucose concentrations were measured by the glucose oxidase technique using an auto analyzer (Roche/Hitachi 902; Roche Diagnostics GmBH, Manheim, Germany). LH, FSH, total testosterone, DHEAS and $\triangle 4 A$ levels were measured by RIA, while SHBG levels were measured by the IRMA, using commercial kits (FSH: Radioisotopic Kit, Nichols Institute Diagnostics, San Juan Capistrano, California, 92675, USA; LH: Radioisotopic Kit, Nichols Institute Diagnostics, San Juan Capistrano, California, 92675, USA; Testo: Radioisotopic Kit, Diagnostic Systems Laboratories, Webster, Texas, USA; $\Delta 4$ A: Radioiso- 
topic Kit, Diagnostic Systems Laboratories, Webster, Texas, USA; DHEAS: Radioisotopic Kit, Diagnostic Systems Laboratories, Webster, Texas, USA; SHBG: Immunoradiometric Assay (IRMA) Kit, Diagnostic Systems Laboratories, Webster, Texas, USA). Serum insulin levels were measured by an enzyme immunoassay (ELISA Kit, Mercodia AB, Uppsala, Sweden). Serum high sensitivity CRP (hsCRP) (mg/l) levels were determined by ELISA (high-sensitivity CRP enzyme immunoassay test kit, LI7500; Linear Chemicals).

The intra-assay coefficients of variation (CV) were $1.5 \%$ for $\mathrm{FSH}, 0.7 \%$ for $\mathrm{LH}, 1.3 \%$ for TT, 5.9 for $\triangle 4 \mathrm{~A}, 9.4 \%$ for DHEAS, $5.8 \%$ for SHBG and $3.8 \%$ for insulin. The average inter-assay $\mathrm{CV}$ were $3.2 \%$ for $\mathrm{FSH}, 1.7 \%$ for $\mathrm{LH}, 2.2 \%$ for Testo, $9.2 \%$ for $\triangle 4 \mathrm{~A}, 12.1 \%$ for DHEAS, $7.8 \%$ for SHBG, $4.4 \%$ for insulin.

\section{Competitive AGE enzyme-linked immunosorbent assay (ELISA)}

The competitive AGE-ELISA procedure was performed as described previously in a PCOS population by Diamanti-Kandarakis et al. ${ }^{10}$

\section{Statistical analysis}

Statistical analysis was performed using the Statistical Package for Social Sciences software (IBM SPSS Statistics for Windows, Version 20.0. Armonk, NY: IBM Corp). Results are presented as means \pm SE for normal variables and as medians (interquartile range) for variables with not normal distribution. The Kolmogorov-Smirnov statistic was performed to test continuous variables for normality. Spearman (rho) or Pearson (r) correlations coefficients were determined for the assessment of the relationship between the examined variables. Repeated measures analysis was performed for comparisons of variables between baseline, 3 and 6 months. In the case of normally distributed variables, repeated measures ANOVA with Bonferroni post-hoc analysis were performed. In the case of non-normal variables, Friedman analysis was performed in addition to pairwise comparisons (Wilcoxon for each pair of groups) adjusting the $\mathrm{p}$ value for each using a Bonferroni multiple comparison procedure. For comparisons among the three treatment groups at baseline, 3 and 6 months we used ANOVA (with Bonferroni posthoc analysis) for normal variables or Kruskal-Wallis (with Mann-Whitney comparisons between the two groups adjusting with Bonferroni multiple comparisons procedure) for non-normal variables. Stepwise multivariate regression analysis was performed in order to define independent predictors of DAGEs. Independent variables included age, treatment regimen $(\mathrm{OCPs}=1$, metformin $=2)$ and all the variables which were found to be correlated in bivariate analyses with the DAGEs. For the results of linear regression analysis the values of beta ( $95 \%$ C.I.) were reported. Normality of residuals was assessed to ensure the feasibility of the regression analysis. Tolerance and variance inflation factor were used for the assessment of potential multicollinearity among the variables retained in the final regression models. A probability value $p<0.05$ was considered statistically significant.

\section{RESULTS}

\section{Baseline characteristics of the patient population}

Baseline anthropometric, metabolic and hormonal parameters of patients are included in Table 1 . The three groups were matched for age $(22 \pm 0.6 \mathrm{yrs}$ in group OCP $1 ; 23.2 \pm 0.6$ yrs in group OCP $2 ; 21.5 \pm 0.5$ yrs in group MET), $\mathrm{W} / \mathrm{H}$ ratio $(0.77 \pm 0.01$ in group OCP1; $0.74 \pm 0.01$ in group OCP2; $0.75 \pm 0.01$ in group MET; $\mathrm{P}>0.05)$ and BMI $\left(21.80 \pm 0.35 \mathrm{~kg} / \mathrm{m}^{2}\right.$ in group OCP $1 ; 22.37 \pm 0.48 \mathrm{~kg} / \mathrm{m}^{2}$ in group OCP $2 ; 23.03 \pm 0.67$ $\mathrm{kg} / \mathrm{m}^{2}$ in group MET; P >0.05) at baseline. Additionally, the three groups did not differ in serum levels of hormonal parameters, AGEs and CRP at baseline, but the MET group had higher baseline HOMA-IR than the OCP1 group (Table 1).

Two patients in group OCP1 [2 out of $40(5 \%)$ ] and four patients in group OCP2 [4 out of $40(10 \%)$ ] were lost to follow-up. Five patients in group MET [5 out of $40(12.5 \%)$ ] dropped out due to gastrointestinal side effects attributed to metformin. Analyses were performed for the 109 women who completed the study (38 in OCP 1 group; 36 in OCP2 group; 35 in MET group). In these women no significant adverse event was reported.

\section{Anthropometric and laboratory parameters during the treatment period}

Anthropometric, metabolic and hormonal parameters at 3 and 6 months of treatment are shown in Table 1. BMI was significantly increased in group 
Table 1. Anthropometric and laboratory parameters of patients who completed the study at baseline, 3 months and 6 months of treatment.

\begin{tabular}{|c|c|c|c|c|c|}
\hline Variable & Time & OCP1 $(n=38)$ & OCP2 $(n=36)$ & MET $(n=35)$ & p value* \\
\hline \multirow[t]{4}{*}{$\mathrm{BMI}\left(\mathrm{kg} / \mathrm{m}^{2}\right)$} & 0 & $21.80 \pm 0.35$ & $22.37 \pm 0.48$ & $23.03 \pm 0.67$ & 0.238 \\
\hline & 3 months & $21.99 \pm 0.36$ & $22.72 \pm 0.48$ & $22.64 \pm 0.68$ & 0.548 \\
\hline & 6 months & $22.28 \pm 0.37$ & $22.68 \pm 0.50$ & $22.44 \pm 0.70$ & 0.867 \\
\hline & $\mathrm{p}$-value $\dagger$ & $<0.001$ & $<0.001$ & 0.001 & \\
\hline \multirow[t]{4}{*}{ HOMA-IR } & 0 & $1.39(1.06)$ & $1.92(1.08)$ & $2.38(1.57)$ & 0.001 \\
\hline & 3 months & 1.89 (1.74) & $2.12(1.53)$ & $1.81(1.61)$ & 0.968 \\
\hline & 6 months & $2.26(1.65)$ & $2.42(1.44)$ & $1.53(0.87)$ & $<0.001$ \\
\hline & $\mathrm{p}$-value $\dagger$ & 0.001 & 0.015 & 0.001 & \\
\hline \multirow[t]{4}{*}{ Testo (nmol/l) } & 0 & $2.95 \pm 0.15$ & $3.00 \pm 0.12$ & $2.85 \pm 0.09$ & 0.681 \\
\hline & 3 months & $1.83 \pm 0.14$ & $1.91 \pm 0.11$ & $2.88 \pm 0.13$ & $<0.001$ \\
\hline & 6 months & $1.61 \pm 0.09$ & $1.99 \pm 0.13$ & $2.32 \pm 0.07$ & $<0.001$ \\
\hline & $\mathrm{p}$-value $\dagger$ & $<0.001$ & $<0.001$ & 0.001 & \\
\hline \multirow[t]{4}{*}{ SHBG (nmol/l) } & 0 & $52.80 \pm 3.70$ & $49.50 \pm 3.70$ & $45.80 \pm 3.40$ & 0.385 \\
\hline & 3 months & $220.20 \pm 15.80$ & $209.30 \pm 11.40$ & $52.50 \pm 4.30$ & $<0.001$ \\
\hline & 6 months & $247.90 \pm 12.90$ & $230.50 \pm 13.00$ & $44.90 \pm 3.00$ & $<0.001$ \\
\hline & $\mathrm{p}$-value $\dagger$ & $<0.001$ & $<0.001$ & 0.049 & \\
\hline \multirow[t]{4}{*}{ FAI } & 0 & $5.82(2.87)$ & $7.48(4.78)$ & $6.40(4.00)$ & 0.42 \\
\hline & 3 months & $0.80(0.69)$ & $0.98(0.67)$ & $5.97(6.42)$ & $<0.001$ \\
\hline & 6 months & $0.66(0.50)$ & $0.88(0.63)$ & $5.71(2.70)$ & $<0.001$ \\
\hline & $\mathrm{p}$-value $\dagger$ & $<0.001$ & $<0.001$ & 0.012 & \\
\hline \multirow[t]{4}{*}{ AGEs (IU/ml) } & 0 & $7.72 \pm 0.33$ & $7.98 \pm 0.27$ & $8.51 \pm 0.29$ & 0.167 \\
\hline & 3 months & $7.32 \pm 0.44$ & $7.93 \pm 0.27$ & $7.91 \pm 0.23$ & 0.325 \\
\hline & 6 months & $7.22 \pm 0.46$ & $7.59 \pm 0.32$ & $6.80 \pm 0.23$ & 0.079 \\
\hline & $\mathrm{p}$-value $\dagger$ & 0.005 & 0.069 & $<0.001$ & \\
\hline \multirow[t]{4}{*}{ CRP (mg/L) } & 0 & $1.36(0.68)$ & $1.09(0.68)$ & $1.34(0.65)$ & 0.841 \\
\hline & 3 months & $1.88(0.72)$ & $1.32(1.07)$ & $1.00(0.28)$ & $<0.001$ \\
\hline & 6 months & $2.63(0.73)$ & $1.93(1.44)$ & $0.75(0.48)$ & $<0.001$ \\
\hline & $\mathrm{p}$-value $\dagger$ & $<0.001$ & $<0.001$ & $<0.001$ & \\
\hline
\end{tabular}

* ANOVA or Kruskal-Wallis test for comparisons between the three groups.

$\dagger$ Repeated measures ANOVA or Friedman test for within group comparisons between 0, 3 and 6 months.

Values are presented as means \pm SE for normal variables or medians (interquartile range) for variables with not normal distribution. To convert testosterone from SI (nmol/l) to conventional units (ng/dl), divide by 0.0347 .

OCP1 $(\mathrm{P}<0.001)$ and group OCP2 $(\mathrm{P}<0.001)$ following 3 and 6 months of treatment compared to baseline, while it was decreased in group MET $(\mathrm{P}=0.001)$. However, BMIs did not differ significantly between the three groups at 3 months $(\mathrm{P}=0.548)$ and 6 months $(\mathrm{P}=0.867)$.

\section{Changes in atherogenic markers during the treatment period}

At 6 months of treatment serum AGEs were significantly decreased as compared to baseline values in group OCP1 $(\mathrm{P}=0.005)$ and group MET $(\mathrm{P}<0.001)$, while there was a marginal decrease in group OCP2 $(\mathrm{P}=0.069)$ (Figure 1).

The percent decrease of serum AGEs over the 6-month period of treatment (DAGEs) was significantly greater in group MET than in groups OCP1 and OCP2 (Group OCP1 vs. group MET: $\mathrm{P}=0.022$; Group OCP2 vs. group MET: $\mathrm{P}=0.001$ ) (Figure 2).

CRP was increased in groups OCP1 $(\mathrm{P}<0.001)$ and $\mathrm{OCP} 2(\mathrm{P}<0.001)$, while it was decreased in group MET $(\mathrm{P}<0.001)$ (Figure 3$)$. The increase of CRP during 6 months was significantly greater with the 


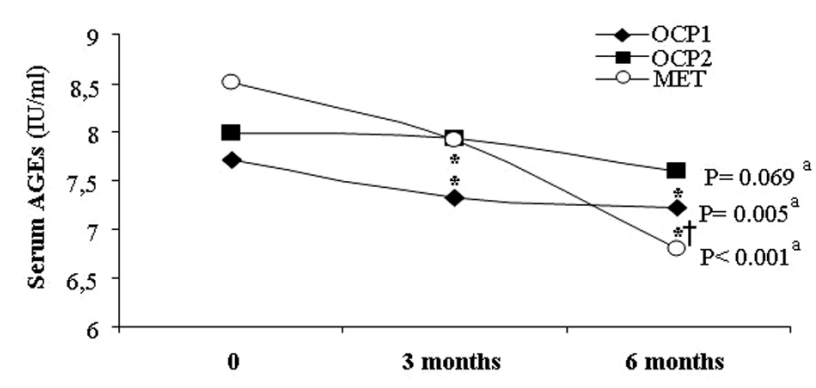

Figure 1. Serum AGEs levels at baseline, 3 months and 6 months of treatment.

* $\mathrm{P}<0.05$ vs baseline in the same group (Wilcoxon test for within group comparison between 0 and 3 or 6 months, respectively, and adjustment with Bonferroni multiple comparison procedure)

$\dagger \mathrm{P}<0.05$ vs 3 months in the same group (Wilcoxon test for within group comparison between 3 and 6 months and adjustment with Bonferroni multiple comparison procedure)

${ }^{a}$ Friedman test for within group comparisons between 0,3 and 6 months.

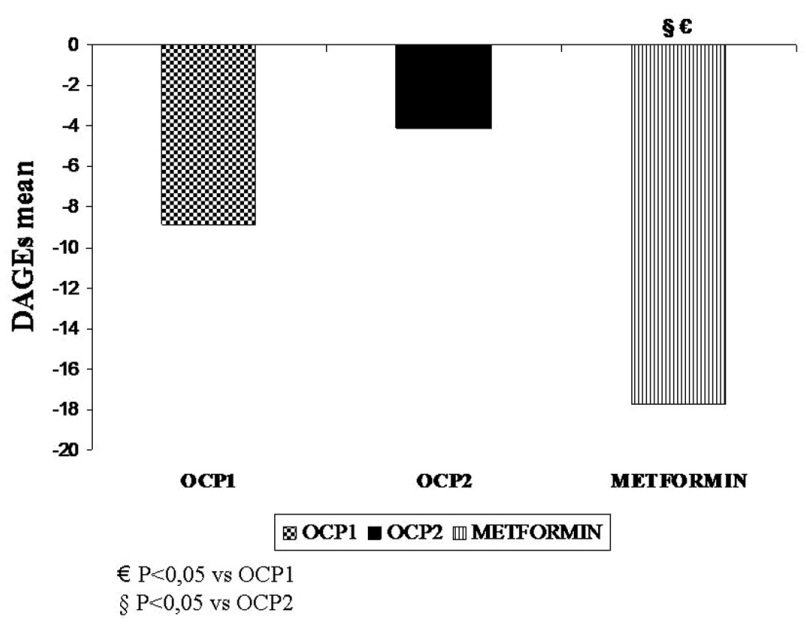

Figure 2. The percent change of serum AGEs (DAGEs) at 6 months of treatment.

CPA pill than with the DRSP pill $(\mathrm{P}=0.003)$.

\section{Correlations of serum AGEs and DAGEs with other variables}

In the total population (three groups as a whole) the change of serum AGEs during the 6-month period (DAGEs) was negatively correlated with baseline BMI $(\mathrm{r}=-0.274, \mathrm{p}=0.004)$, while DCRP was correlated negatively with DTESTO $(\mathrm{r}=-0.506)$ and positively with DSHBG $(\mathrm{r}=0.431)$, DBMI $(\mathrm{rho}=0.494)$ and DHOMA (rho $=0.459)$.

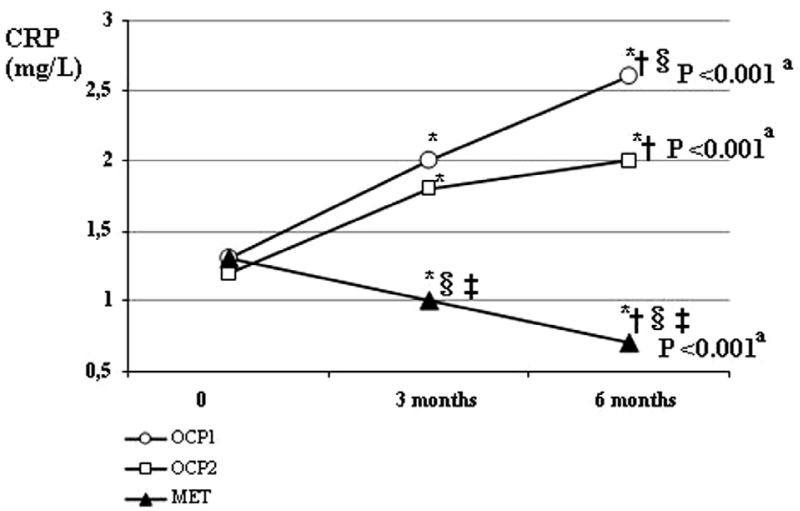

Figure 3. CRP value at baseline, 3 months and 6 months of treatment.

${ }^{*} \mathrm{P}<0.05$ vs baseline in the same group (Wilcoxon test for within group comparison between 0 and 3 or 6 months, respectively, and adjustment with Bonferroni multiple comparison procedure)

$\dagger \mathrm{P}<0.05$ vs 3 months in the same group (Wilcoxon test for within group comparison between 3 and 6 months and adjustment with Bonferroni multiple comparison procedure)

$\ddagger \mathrm{P}<0.05$ vs OCP1 group at the same time (Mann-Whitney test for between two group comparison at the same time and adjustment with Bonferroni multiple comparisons procedure) $\S \mathrm{P}<0.05$ vs OCP2 group at the same time (Mann-Whitney test for between two group comparison at the same time and adjustment with Bonferroni multiple comparisons procedure)

${ }^{a}$ Friedman test for within group comparisons between 0,3 and 6 months.

When subgroup analysis was performed, DAGEs were significantly correlated with age $(\mathrm{r}=0.27)$, baseline BMI $(\mathrm{r}=-0.35)$, baseline TESTO $(\mathrm{r}=-0.35)$, DBMI $(r h o=-0.25)$, DTESTO $(r=0.27)$ and DFAI $(\mathrm{rho}=0.28)$ in women treated with OCPs (OCP1 group $+\mathrm{OCP} 2$ group). In the group treated with metformin, none of the above correlations was observed.

There was no correlation between HOMA-IR and AGEs or DAGEs either in the total group or in the subgroup analysis.

Multivariate regression analysis was performed to examine which parameters were independently associated with DAGEs (dependent variable). Treatment regimen, age, baseline BMI and the change of BMI during the 6-month period (DBMI) were introduced as independent variables. DAGEs was independently associated $\left(\mathrm{R}^{2}=0.13\right)$ with metformin treatment [beta $(95 \% \mathrm{CI})=-8.58(-15.3$ to -1.86$)]$ as well as with a higher baseline BMI [beta $(95 \% \mathrm{CI})=$ 
$-1.23(-2.23$ to -0.23$)]$. Variance inflation factor was 1.5 (tolerance 0.6 ), which ensures the lack of collinearity in the model.

\section{DISCUSSION}

This study is the first to compare the effects of metformin and two different OCPs on serum AGEs in lean women with PCOS. Metformin as well as the $\mathrm{OCP}$ containing cyproterone acetate were effective in reducing serum AGEs, while the OCP containing drospirenone had a marginal lowering effect on this marker. Most importantly, metformin compared to OCPs was associated with a greater degree of AGEs reduction.

The treatment-induced changes in AGEs levels may have significant implications, since circulating AGEs are increased in women with $\mathrm{PCOS}^{2}$ and these molecules are known to exert direct cardiovascular actions promoting the atherogenic process. ${ }^{3}$

The OCP-induced decrease in serum AGEs levels was correlated with the percent reduction of androgen levels, which was significantly greater with either OCP than with metformin. The direct association of serum AGEs with serum testosterone levels has been previously reported by studies in women with PCOS, independently of BMI and insulin resistance. ${ }^{2,10}$ Thus, the androgen lowering effect of OCPs may play a role in the reduction of serum AGEs.

However, treatment with OCPs had adverse effects on other metabolic aspects and cardiovascular risk factors. In the OCP-treated group, BMI, HOMA-IR and $\mathrm{CRP}$ were increased during the 6-month period of treatment. These findings are consistent with earlier studies in women with PCOS. ${ }^{11-14}$ In view of the mixed effects of OCPs on atherogenic markers, the benefit-to-risk ratio of this treatment should be carefully assessed focusing not only on patients' characteristics, but also specifically on the targeted atherogenic molecule. Even among lean women with PCOS, OCPs may not be the treatment of choice in some instances. In particular, metformin rather than OCPs may be the preferred option when treatment is aimed at improving atherogenic markers, including a greater reduction of serum AGEs and a decrease of CRP.
This study evaluates common therapeutic options in women with PCOS by reconsidering and prioritizing the goals of treatment. Since inflammation and the process of glycooxidation may play a central role in the pathophysiology of this complex syndrome, ${ }^{2,4,15}$ both AGEs and CRP could be important targets in PCOS therapy. It can be suggested that pre- and posttreatment atherogenic markers may be included in the criteria of treatment choice and follow-up. This approach takes into account the recognized linkage of PCOS with increased cardiovascular risk. ${ }^{16}$ In addition, inflammatory molecules and endogenous AGEs may also contribute to the pathophysiology of endocrine and reproductive aberrations in PCOS., 2,4,15,17

The net impact of treatment on the cardiovascular profile of PCOS women may depend on the type of OCP. The OCPs used in the present study differ in the type of the progestin component as well as the dose of EE per tablet, with higher EE dose in the CPA pill compared to the DRSP pill. In particular, when comparing the two OCPs, the DRSP pill was associated with a lower degree of BMI and CRP increase as compared to the CPA pill.

The vascular bed appears to be a delicate target for estrogens, since estrogen may be either beneficial or harmful in a dose-dependent manner, which might be relevant for effects of OCPs in PCOS treatment. In experimental models the therapeutic range of estrogens appears to be narrow. Most strikingly, their beneficial effect on vascular tissue was shown to be diminished at larger doses, which yielded high, but still physiologically relevant, plasma estradiol levels. ${ }^{19}$ In this context, OCPs with higher estrogen dose may increase tissue estrogen levels beyond the narrow beneficial range. In the present study, the higher amount of estrogen in the CPA pill compared to the DRSP pill may play a part in the greater increase of CRP with the former.

Furthermore experimental data suggest an interaction between CRP and the Receptor for AGEs (RAGE) via activation of estrogen receptor-alpha. ${ }^{20,21}$ More specifically, 17-beta estradiol was shown to enhance RAGE gene transcription, which stimulates inflammatory cascades leading to overproduction of inflammatory molecules like CRP. ${ }^{22}$ The potential upregulation of RAGE by estrogens in PCOS, which 
is characterized by excess of AGEs,${ }^{15}$ may exacerbate the proinflammatory state, further potentiating adverse cardiovascular consequences. ${ }^{23}$

However, the two OCPs used in the present study did not appear to induce a generalized exacerbation of the oxidative/inflammatory state, since serum AGEs were significantly or marginally decreased in the OCP groups. Thus, the increase of CRP may reflect firstpass hepatic effect of the estrogenic component on hepatic proteins rather than a heightened inflammatory state. ${ }^{24}$ This concept may also be supported by the significant direct correlation of the percent increase of CRP with the percent increase of SHBG, which is also produced in the liver. Therefore, the higher content of EE of the CPA pill compared to the DRSP pill may account for the greater percent increase of CRP with the former drug due to exposure of the liver to a higher concentration of estrogen.

Additionally, the different progestin components of the two OCPs may partly account for different effects on metabolic and cardiovascular aspects. Drospirenone, the progestin component of the DRSP pill, is devoid of androgenic or glucocorticoid activity and has higher antimineralocorticoid potency compared to CPA..$^{25}$ The potential benefits offered by this superior pharmacological profile of DRSP may translate into a less aggravating effect on body weight and inflammation, as shown in our study. From a therapeutic perspective, these data may prove to be helpful in selecting the more beneficial or the less harmful OCP for the treatment of PCOS patients.

Regardless of the mechanism by which OCPs increase CRP, this observation may have important clinical implications. Since prospective, observational studies indicated that CRP is an independent predictor of future cardiovascular events, even in healthy women on hormone replacement therapy, ${ }^{26}$ the elevated CRP induced by OCPs may have clinical significance. CRP appears to be more than a marker predicting cardiovascular events. It has also been shown that CRP exerts a wide array of proatherogenic effects, including inhibition of the production of the vasodilator nitric oxide in human endothelial cells, promotion of monocyte chemotaxis and endothelial shedding of adhesion molecules. ${ }^{27}$ It remains to be established whether the CRP elevation induced by treatment with OCPs directly promotes atherosclerosis in women with PCOS. Moreover, it should be explored how the elevation of CRP interacts with the decrease of AGEs, both induced by OCP treatment, and what the net impact of this interaction is on the atherogenic process.

However, metformin appears to be superior to OCPs in ameliorating the atherogenic profile with a global beneficial impact on cardiometabolic markers, including HOMA-IR, CRP and AGEs. The AGEs-lowering effect of metformin appears to be independent of its effects on androgens, insulin resistance and inflammation. In particular, although the patients treated with metformin had higher baseline HOMA-IR, the percent decrease of AGEs was not correlated with baseline HOMA-IR either in the total population or in subgroups. Moreover, the betweengroup difference of HOMA-IR cannot account for any of our results, since the higher HOMA-IR in the group of metformin would be associated with a worse proinflammatory state. However, treatment with metformin resulted in lower CRP levels and a greater percent decrease of AGEs compared with the other groups. Thus, metformin may act on CRP production and AGEs synthesis or clearance through direct mechanisms in PCOS women. Earlier data suggest that metformin reacts with AGEs extracellularly leading to the formation of inactive condensation products. In addition, metformin appears to have moderate breaking properties on established AGE-protein crosslinks. ${ }^{28-30}$ Thus, metformin may be a multistage inhibitor of glycation and an AGE-protein crosslink breaker, independently of its effects on glucose metabolism, insulin signalling and inflammation.

Our study has limitations. The small sample size may lack the power to detect potential additional effects of treatment on outcome measures and may obscure any modest correlations between the studied parameters. In addition, changes in cardiovascular risk markers cannot accurately predict any impact on clinical outcomes. Furthermore, longer duration of treatment may be required for its optimal efficacy. More specifically, a recent study showed that treatment for 12 months may be required for CPA to achieve its maximal antiandrogenic effects in women with PCOS. ${ }^{31}$ However, the effects of three different types of OCPs (containing CPA, desogestrel and DRSP) 
on metabolic aspects (BMI and insulin resistance indices) did not differ between 6 and 12 months of treatment. ${ }^{31}$ Future studies should clarify the effects of greater duration of OCP treatment on atherogenic markers in women with PCOS.

Understanding the pathophysiology of PCOS has rearranged the priorities in its therapeutic approach, placing weight on the amelioration of cardiovascular risk associated with the syndrome. Overall, the present study adds to the evidence that OCPs and metformin may have differential effects on specific cardiovascular risk factors associated with PCOS. In lean women with PCOS, metformin appears to be superior to OCPs in metabolic and cardiovascular aspects. However, both these treatments may have strengths and drawbacks in treating different aspects of this multifaceted syndrome. Thus, clinicians should remain alert to detect adverse effects, when prescribing any medication for PCOS women, as they do for other heterogeneous disorders, like type 2 diabetes.

\section{FUNDING}

This research did not receive any specific grant from any funding agency in the public, commercial or not-for-profit sector.

\section{CONFLICT OF INTEREST}

The authors have nothing to declare.

\section{REFERENCES}

1. Diamanti-Kandarakis E, Dunaif A, 2012 Insulin resistance and the polycystic ovary syndrome revisited: an update on mechanisms and implications. Endocr Rev 33: 981-1030.

2. Diamanti-Kandarakis E, Katsikis I, Piperi C, et al, 2008 Increased serum advanced glycation end-products is a distinct finding in lean women with polycystic ovary syndrome (PCOS). Clin Endocrinol (Oxf) 69: 634-641.

3. Vlassara H, 2005 Advanced glycation in health and disease: role of the modern environment. Ann N Y Acad Sci 1043: 452-460.

4. Diamanti-Kandarakis E, Piperi C, Patsouris E, et al, 2007 Immunohistochemical localization of advanced glycation end-products (AGEs) and their receptor (RAGE) in polycystic and normal ovaries. Histochem Cell Biol 127: 581-589.

5. Baillargeon JP, McClish DK, Essah PA, Nestler JE,
2005 Association between the current use of low-dose oral contraceptives and cardiovascular arterial disease: a metaanalysis. J Clin Endocrinol Metab 90: 3863-3870.

6. Merz CN, Johnson BD, Berga S, Braunstein G, Reis SE, Bittner V, WISE Study Group, 2006 Past oral contraceptive use and angiographic coronary artery disease in postmenopausal women: data from the National Heart, Lung, and Blood Institute-sponsored Women's Ischemia Syndrome Evaluation. Fertil Steril 85: 1425-1431.

7. Diamanti-Kandarakis E, Paterakis T, Alexandraki K, et al, 2006 Indices of low-grade chronic inflammation in polycystic ovary syndrome and the beneficial effect of metformin. Hum Reprod 21: 1426-1431.

8. Diamanti-Kandarakis E, Alexandraki K, Piperi C, et al, 2007 Effect of metformin administration on plasma advanced glycation end product levels in women with polycystic ovary syndrome. Metabolism 56: 129-134.

9. Zawadski JK, Dunaif A 1992 Diagnostic criteria for polycystic ovary syndrome: Towards a rational approach. In: Dunaif A, Givens JR, Haseltine FP, Merriam GE (eds) Polycystic Ovary Syndrome (Current Issues in Endocrinology and Metabolism), Blackwell Scientific Inc, Boston; p, 377.

10. Diamanti-Kandarakis E, Piperi C, Kalofoutis A, Creatsas G, 2005 Increased levels of serum advanced glycation end-products in women with polycystic ovary syndrome. Clin Endocrinol (Oxf) 62: 37-43.

11. Morin-Papunen LC, Vauhkonen I, Koivunen RM, Ruokonen A, Martikainen HK, Tapanainen JS, 2003 Metformin versus ethinyl estradiol-cyproterone acetate in the treatment of nonobese women with polycystic ovary syndrome: a randomized study. J Clin Endocrinol Metab 88: 148-156.

12. Nader S, Diamanti-Kandarakis E, 2007 Polycystic ovary syndrome, oral contraceptives and metabolic issues: new perspectives and a unifying hypothesis. Hum Reprod 22: 317-322.

13. Mastorakos G, Koliopoulos C, Deligeoroglou E, Diamanti-Kandarakis E, Creatsas G, 2006 Effects of two forms of combined oral contraceptives on carbohydrate metabolism in adolescents with polycystic ovary syndrome Fertil Steril 85: 420-427.

14. Teede HJ, Meyer C, Hutchison SK, Zoungas S, McGrath BP, Moran LJ, 2010 Endothelial function and insulin resistance in polycystic ovary syndrome: the effects of medical therapy. Fertil Steril 93: 184-191.

15. González F, 2012 Inflammation in Polycystic Ovary Syndrome: underpinning of insulin resistance and ovarian dysfunction. Steroids 77: 300-305.

16. Christakou C, Diamanti-Kandarakis E, 2013 Structural, biochemical and non-traditional cardiovascular risk markers in PCOS. Curr Pharm Des 19: 5764-5774.

17. Jabbour HN, Sales KJ, Catalano RD, Norman JE, 2009 Inflammatory pathways in female reproductive health and disease. Reproduction 138: 903-919.

18. Corrado E, Rizzo M, Coppola G, et al, 2010 An update 
on the role of markers of inflammation in atherosclerosis. J Atheroscler Thromb 17: 1-11.

19. Rusa R, Alkayed N, Crain B, et al, 1999 17beta-estradiol reduces stroke injury in estrogen-deficient female animals. Stroke 30: 1665-1670.

20. Mukherjee TK, Reynolds PR, Hoidal JR, 2005 Differential effect of estrogen receptor alpha and beta agonists on the receptor for advanced glycation end product expression in human microvascular endothelial cells. Biochim Biophys Acta 1745: 300-309.

21. Mukhopadhyay S, Mukherjee TK, 2005 Bridging advanced glycation end product, receptor for advanced glycation end product and nitric oxide with hormonal replacement/estrogen therapy in healthy versus diabetic postmenopausal women: a perspective. Biochim Biophys Acta 1745: 145-155.

22. Kislinger T, Tanji N, Wendt T, et al, 2001 Receptor for advanced glycation end products mediates inflammation and enhanced expression of tissue factor in vasculature of diabetic apolipoprotein E-null mice. Arterioscler Thromb Vasc Biol 21: 905-910.

23. Schmidt AM, Yan SD, Wautier JL, Stern D, 1999 Activation of receptor for advanced glycation end products: a mechanism for chronic vascular dysfunction in diabetic vasculopathy and atherosclerosis. Circ Res 84: 489-497.

24. Vongpatanasin W, Tuncel M, Wang Z, Arbique D, Meh$\operatorname{rad}$ B, Jialal I, 2003 Differential effects of oral versus transdermal estrogen replacement therapy on C-reactive protein in postmenopausal women. J Am Coll Cardiol 41: 1358-1363.

25. Palacios S, Foidart JM, Genazzani A, 2006 Advances in hormone replacement therapy with drospirenone, a unique progestogen with aldosterone receptor antagonism. Maturitas 55: 297-307.

26. Ridker PM, Rifai N, Rose L, Buring JE, Cook NR, 2002 Comparison of C-reactive protein and low-density lipoprotein cholesterol levels in the prediction of first cardiovascular events. N Engl J Med 347: 1557-1565.

27. Packard R, Libby P, 2008 Inflammation in Atherosclerosis: From Vascular Biology to Biomarker Discovery and Risk Prediction. Clin Chem 54: 24-38.

28. Beisswenger PJ, Ruggiero-Lopez D, 2003 Metformin inhibition of glycation processes. Diabetes Metab 29(4 Pt 2): 6S95-103.

29. Tanaka Y, Iwamoto H, Onuma T, Kawamori R, 1997 Inhibitory effect of metformin on formation of advanced glycation end products. Curr Ther Res 58: 693-697.

30. Tanaka Y, Uchino H, Shimizu T, et al, 1999 Effect of metformin on advanced glycation end product formation and peripheral nerve function in streptozotocin-induced diabetic rats. Eur J Pharmacol 376: 17-22.

31. Bhattacharya SM, Jha A, 2012 Comparative study of the therapeutic effects of oral contraceptive pills containing desogestrel, cyproterone acetate, and drospirenone in patients with polycystic ovary syndrome. Fertil Steril 98: 1053-1059. 